\title{
Hilbert Transforms Associated with Dunkl-Hermite Polynomials ${ }^{\star}$
}

\author{
Néjib BEN SALEM and Taha SAMAALI
}

Department of Mathematics, Faculty of Sciences of Tunis, Campus Universitaire, 2092 Tunis, Tunisia

E-mail: Nejib.BenSalem@fst.rnu.tn

Received October 14, 2008, in final form March 12, 2009; Published online March 25, 2009 doi:10.3842/SIGMA.2009.037

\begin{abstract}
We consider expansions of functions in $L^{p}\left(\mathbb{R},|x|^{2 k} d x\right), 1 \leq p<+\infty$ with respect to Dunkl-Hermite functions in the rank-one setting. We actually define the heat-diffusion and Poisson integrals in the one-dimensional Dunkl setting and study their properties. Next, we define and deal with Hilbert transforms and conjugate Poisson integrals in the same setting. The formers occur to be Calderón-Zygmund operators and hence their mapping properties follow from general results.
\end{abstract}

Key words: Dunkl operator; Dunkl-Hermite functions; Hilbert transforms; conjugate Poisson integrals; Calderón-Zygmund operators

2000 Mathematics Subject Classification: 42A50; 42C10

\section{Introduction}

The study of Dunkl operators has known a considerable growth during the last two decades due to their relevance in mathematical physics and since they give the way to built a parallel to the theory of spherical Harmonics based on finite root systems and depending on a set of real parameters. In this spirit, the Hilbert transform, a basic tool in signal processing and in Fourier and harmonic analysis as well, may be defined by means of partial derivatives, so that, since the commutative algebra of Dunkl operators generalize the one of partial derivatives, it is natural to extend the study of Hilbert transforms and connected topics as heat diffusion, Poisson integrals and others to the Dunkl setting. In this work, we start with investigating the rank-one case, that is why we sketch some facts that are subsequently needed. Let $k$ be a nonnegative parameter and let $T_{k}$ be the Dunkl operator acting on smooth functions $f$ as

$$
T_{k} f(x)=f^{\prime}(x)+k \frac{f(x)-f(-x)}{x}, \quad f \in C^{1}(\mathbb{R}) .
$$

To that operator is associated the so-called Dunkl-Hermite operator on $\mathbb{R}$ denoted $L_{k}$ and defined by

$$
L_{k}=T_{k}^{2}-x^{2} .
$$

Its spectral decomposition is given by the Dunkl-Hermite functions $h_{n}^{k}$ defined by

$$
h_{n}^{k}(x)=e^{-\frac{x^{2}}{2}} H_{n}^{k}(x),
$$

where $H_{n}^{k}$ are the generalized Hermite polynomials which we call the Dunkl-Hermite polynomials as in [3], namely (see [8])

$$
L_{k} h_{n}^{k}(x)=-(2 n+2 k+1) h_{n}^{k}(x) .
$$

\footnotetext{
${ }^{\star}$ This paper is a contribution to the Special Issue on Dunkl Operators and Related Topics. The full collection is available at http://www.emis.de/journals/SIGMA/Dunkl_operators.html
} 
Recall also that $H_{n}^{k}$ were introduced in [7] and studied by Rösler in [8], whence

$$
\begin{aligned}
& H_{2 n}^{k}(x)=(-1)^{n} \sqrt{\frac{n !}{\Gamma\left(n+k+\frac{1}{2}\right)}} L_{n}^{k-\frac{1}{2}}\left(x^{2}\right), \\
& H_{2 n+1}^{k}(x)=(-1)^{n} \sqrt{\frac{n !}{\Gamma\left(n+k+\frac{3}{2}\right)}} x L_{n}^{k+\frac{1}{2}}\left(x^{2}\right),
\end{aligned}
$$

where $L_{n}^{\alpha}$ are the Laguerre polynomials of index $\alpha \geq-\frac{1}{2}$, given by

$$
L_{n}^{\alpha}(x)=\frac{1}{n !} x^{-\alpha} e^{x} \frac{d^{n}}{d x^{n}}\left(x^{n+\alpha} e^{-x}\right) .
$$

It is well known that the system $\left\{H_{n}^{k}\right\}_{n \geq 0}$ is complete and orthonormal in $L^{2}\left(\mathbb{R}, e^{-x^{2}}|x|^{2 k} d x\right)$, therefore the system $\left\{h_{n}^{k}\right\}_{n \geq 0}$ is complete and orthonormal in $L^{2}\left(\mathbb{R},|x|^{2 k} d x\right)$.

Hereafter, $L^{p}\left(\mathbb{R},|x|^{2 k} d x\right), 1 \leq p<+\infty$ is the space of measurable functions on $\mathbb{R}$ satisfying

$$
\|f\|_{k, p}:=\left(\int_{\mathbb{R}}|f(x)|^{p}|x|^{2 k} d x\right)^{\frac{1}{p}}<+\infty
$$

and $f$ belongs to $L^{p}\left(\mathbb{R},|x|^{2 k} d x\right), 1 \leq p<+\infty$, unless mentioned. For a given $f$, one defines the heat-diffusion integral $G_{k}(f)$ by

$$
G_{k}(f)(t, x)=\sum_{n=0}^{+\infty} e^{-t(2 n+2 k+1)} a_{n}^{k}(f) h_{n}^{k}(x), \quad t>0, \quad x \in \mathbb{R},
$$

where

$$
a_{n}^{k}(f)=\int_{\mathbb{R}} f(t) h_{n}^{k}(t)|t|^{2 k} d t .
$$

We shall establish that $G_{k}(f)$ possesses the following integral representation

$$
G_{k}(f)(t, x)=\int_{\mathbb{R}} P_{k}(t, x, y) f(y)|y|^{2 k} d y
$$

where

$$
P_{k}(t, x, y)=\sum_{n=0}^{+\infty} e^{-t(2 n+2 k+1)} h_{n}^{k}(x) h_{n}^{k}(y) .
$$

We shall prove that $G_{k}(f)(t, \cdot), t>0$, satisfies

$$
\left\|G_{k}(f)(t, \cdot)\right\|_{k, p} \leq(\cosh (2 t))^{-\left(k+\frac{1}{2}\right)}\|f\|_{k, p} .
$$

Next, we define the Poisson integral $F_{k}(f)$ by

$$
F_{k}(f)(t, x)=\sum_{n=0}^{+\infty} e^{-t \sqrt{2 n+2 k+1}} a_{n}^{k}(f) h_{n}^{k}(x), \quad t>0, \quad x \in \mathbb{R} .
$$

We shall establish that $F_{k}(f)$ possesses the following integral representation

$$
F_{k}(f)(t, x)=\int_{\mathbb{R}} f(y) A_{k}(t, x, y)|y|^{2 k} d y
$$


where

$$
A_{k}(t, x, y)=\frac{t}{\sqrt{4 \pi}} \int_{0}^{+\infty} P_{k}(u, x, y) u^{-\frac{3}{2}} e^{-\frac{t^{2}}{4 u}} d u
$$

is the Poisson kernel associated with $\left\{h_{n}^{k}\right\}_{n \geq 0}$. We shall show that $F_{k}(f)(t, \cdot) \in L^{p}\left(\mathbb{R},|x|^{2 k} d x\right)$ and

$$
\left\|F_{k}(f)(t, \cdot)\right\|_{k, p} \leq 2^{k+\frac{1}{2}} e^{-t \sqrt{2 k+1}}\|f\|_{k, p} .
$$

Also, we define the Hilbert transforms associated with the Dunkl-Hermite operators formally by

$$
\mathcal{H}_{k}^{ \pm}=\left(T_{k} \pm x\right)\left(-L_{k}\right)^{-\frac{1}{2}} .
$$

We write $f \sim \sum_{n=0}^{+\infty} a_{n}^{k}(f) h_{n}^{k}$, to say that the last series represents the expansions of $f$ with respect to $\left\{h_{n}^{k}\right\}_{n \geq 0}$. Note, that if $f \sim \sum_{n=0}^{+\infty} a_{n}^{k}(f) h_{n}^{k}$, then again formally,

$$
\mathcal{H}_{k}^{+} f \sim \sum_{n=0}^{+\infty} a_{n}^{k}(f) \frac{\theta(n, k)}{\sqrt{2 n+2 k+1}} h_{n-1}^{k}, \quad \mathcal{H}_{k}^{-} f \sim-\sum_{n=0}^{+\infty} a_{n}^{k}(f) \frac{\theta(n+1, k)}{\sqrt{2 n+2 k+1}} h_{n+1}^{k},
$$

where

$$
\theta(n, k)= \begin{cases}\sqrt{2 n} & \text { if } n \text { is even, } \\ \theta(n, k)=\sqrt{2 n+4 k} & \text { if } n \text { is odd }\end{cases}
$$

here and also later on, we use the convention that $h_{n-1}^{k}=0$ if $n=0$.

We shall see that

$$
\mathcal{H}_{k}^{ \pm} f(x)=\lim _{\epsilon \longrightarrow 0} \int_{|x-y|>\epsilon} f(y) R_{k}^{ \pm}(x, y)|y|^{2 k} d y,
$$

exist for almost every $x$, where $R_{k}^{ \pm}(x, y)$ are appropriate kernels. Next, we shall prove that the operators $\mathcal{H}_{k}^{ \pm}$are bounded on $L^{p}\left(\mathbb{R},|x|^{2 k} d x\right)$.

Finally, we use the Dunkl-Hermite functions to define the conjugate Poisson integrals $f_{k}^{ \pm}(t, x)$ by

$$
\begin{aligned}
& f_{k}^{+}(t, x)=\sum_{n=0}^{+\infty} e^{-t \sqrt{2 n+2 k+1}} a_{n}^{k}(f) \frac{\theta(n, k)}{\sqrt{2 n+2 k+1}} h_{n-1}^{k}(x), \\
& f_{k}^{-}(t, x)=\sum_{n=0}^{+\infty} e^{-t \sqrt{2 n+2 k+1}} a_{n}^{k}(f) \frac{\theta(n+1, k)}{\sqrt{2 n+2 k+1}} h_{n+1}^{k}(x) .
\end{aligned}
$$

We shall establish that $f_{k}^{ \pm}(t, x)$ possesses the integral representations

$$
f_{k}^{+}(t, x)=\int_{\mathbb{R}} Q_{k}(t, x, y) f(y)|y|^{2 k} d y, \quad f_{k}^{-}(t, x)=\int_{\mathbb{R}} M_{k}(t, x, y) f(y)|y|^{2 k} d y,
$$

where $Q_{k}(t, x, \cdot)$ and $M_{k}(t, x, \cdot)$ are kernels expressed in terms of the Dunkl kernel $E_{k}(x, y)$ which is the eigenfunction of the Dunkl operator $T_{k}$.

We point out that recently (see [6]) A. Nowak and K. Stempak have studied Riesz transforms for the Dunkl harmonic oscillator in the rank-one case.

We conclude this introduction by giving the organization of this paper. In the second section, we define the heat-diffusion integral $G_{k}(f)$ and the Poisson integral $F_{k}(f)$ and give the integral representations of $G_{k}(f)$ and $F_{k}(f)$. In the third section, we deal with the Hilbert transforms $\mathcal{H}_{k}^{ \pm}$ and prove that these operators are of the strong type $(p, p), 1<p<+\infty$. The remaining part is concerned with the study of the conjugate Poisson. 


\section{Heat-diffusion and Poisson integrals}

\subsection{Heat-diffusion}

With the help of the Dunkl-Hermite functions introduced in the previous section, we define and study the heat-diffusion in the Dunkl setting. As the Dunkl-Hermite polynomials are expressed in terms of Laguerre polynomials, using Lemma 1.5.4 in [12], we have the following limiting behavior of $\left\|h_{n}^{k}\right\|_{k, p}$, with respect to $n$.

Proposition 1. For $1 \leq p \leq 4$, we have

$$
\begin{aligned}
& \left\|h_{2 n}^{k}\right\|_{k, p} \sim \begin{cases}n^{-\frac{1}{4}+\frac{1}{2 p}+k\left(\frac{1}{p}-\frac{1}{2}\right)}, & \text { if } k(p-2)<1, \\
n^{-\frac{1}{4}-\frac{1}{2 p}+k\left(\frac{1}{2}-\frac{1}{p}\right)}, & \text { if } k(p-2)>1,\end{cases} \\
& \left\|h_{2 n+1}^{k}\right\|_{k, p} \sim \begin{cases}n^{-\frac{1}{4}+\frac{1}{2 p}+k\left(\frac{1}{p}-\frac{1}{2}\right)}, & \text { if } k(p-2)<1, \\
n^{-\frac{1}{4}-\frac{1}{2 p}+k\left(\frac{1}{2}-\frac{1}{p}\right)}, & \text { if } k(p-2)>1 .\end{cases}
\end{aligned}
$$

For $p>4$, we have

$$
\begin{aligned}
& \left\|h_{2 n}^{k}\right\|_{k, p} \sim \begin{cases}n^{-\frac{1}{12}-\frac{1}{6 p}+k\left(\frac{1}{p}-\frac{1}{2}\right)}, & \text { if } k(p-2) \leq \frac{1}{3}+\frac{p}{6}, \\
n^{-\frac{1}{4}-\frac{1}{2 p}+k\left(\frac{1}{2}-\frac{1}{p}\right)}, & \text { if } k(p-2)>\frac{1}{3}+\frac{p}{6},\end{cases} \\
& \left\|h_{2 n+1}^{k}\right\|_{k, p} \sim \begin{cases}n^{-\frac{1}{12}-\frac{1}{6 p}+k\left(\frac{1}{p}-\frac{1}{2}\right)}, & \text { if } k(p-2) \leq \frac{1}{3}+\frac{p}{6}, \\
n^{-\frac{1}{4}-\frac{1}{2 p}+k\left(\frac{1}{2}-\frac{1}{p}\right)}, & \text { if } k(p-2)>\frac{1}{3}+\frac{p}{6} .\end{cases}
\end{aligned}
$$

Proposition 2. There exists a positive constant $C$ such that

$$
\left\|h_{n}^{k}\right\|_{\infty} \leq C n^{\frac{k}{2}-\frac{1}{12}} .
$$

Proof. Using the fact that

$$
H_{2 n}^{k}=\frac{\sqrt{n ! \Gamma\left(n+k+\frac{1}{2}\right)}}{(2 n) ! \Gamma\left(k+\frac{1}{2}\right)} V_{k}\left(H_{2 n}\right)
$$

(see [7]), where $\left\{H_{2 n}\right\}_{n \geq 0}$ is the set of classical Hermite polynomials, and $V_{k}$ is the intertwining operator between $T_{k}$ and the usual derivative $\frac{d}{d x}$ given by

$$
V_{k}(f)(x)=\frac{2^{-2 k} \Gamma(2 k+1)}{\Gamma(k) \Gamma(k+1)} \int_{-1}^{1} f(x t)\left(1-t^{2}\right)^{k-1}(1+t) d t,
$$

we deduce that

$$
\left\|h_{2 n}^{k}\right\|_{\infty} \leq C \frac{2^{n} \sqrt{n ! \Gamma\left(n+k+\frac{1}{2}\right)}}{\sqrt{(2 n) !} \Gamma\left(k+\frac{1}{2}\right)}\left\|h_{2 n}\right\|_{\infty},
$$

where $\left\{h_{2 n}\right\}_{n \geq 0}$ is the classical Hermite-functions. In view of the following estimate

$$
\left\|h_{n}\right\|_{\infty} \leq C n^{-\frac{1}{12}}
$$

given in [4, Lemma 2.1], using Stirling's formula, we can deduce easily the result.

In the same way, we have

$$
H_{2 n+1}^{k}=\frac{\sqrt{n ! \Gamma\left(n+k+\frac{3}{2}\right)}}{(2 n+1) ! \Gamma\left(k+\frac{1}{2}\right)} V_{k}\left(H_{2 n+1}\right),
$$

where $\left\{H_{2 n+1}\right\}_{n \geq 0}$ is the classical Hermite polynomials, we obtain the result as above. 
Let

$$
a_{n}^{k}(f)=\int_{\mathbb{R}} f(t) h_{n}^{k}(t)|t|^{2 k} d t .
$$

Using the fact that $h_{n}^{k} \in L^{p^{\prime}}\left(\mathbb{R},|x|^{2 k} d x\right)$, where $p^{\prime}$ is the conjugate exponent of $p$, and Hölder's inequality, we deduce that

$$
\left|a_{n}^{k}(f)\right| \leq\|f\|_{k, p}\left\|h_{n}^{k}\right\|_{k, p^{\prime}}
$$

In view of Propositions 1 and 2, we have the following.

Proposition 3. The series

$$
\sum_{n=0}^{+\infty} e^{-t(2 n+2 k+1)} a_{n}^{k}(f) h_{n}^{k}(x), \quad t>0,
$$

converges uniformly in $x \in \mathbb{R}$.

Definition 1. We define the heat-diffusion integral of $f$ by

$$
G_{k}(f)(t, x)=\sum_{n=0}^{+\infty} e^{-t(2 n+2 k+1)} a_{n}^{k}(f) h_{n}^{k}(x), \quad t>0, \quad x \in \mathbb{R} .
$$

Proposition 4. The heat-diffusion integral $G_{k}(f)$ possesses the following integral representation

$$
G_{k}(f)(t, x)=\int_{\mathbb{R}} P_{k}(t, x, y) f(y)|y|^{2 k} d y,
$$

where

$$
P_{k}(t, x, y)=\sum_{n=0}^{+\infty} e^{-t(2 n+2 k+1)} h_{n}^{k}(x) h_{n}^{k}(y) .
$$

Proof. We obtain an integral form of $G_{k}(f)$ by writing

$$
\begin{aligned}
G_{k}(f)(t, x) & =\sum_{n=0}^{+\infty} e^{-t(2 n+2 k+1)} h_{n}^{k}(x) \int_{\mathbb{R}} f(y) h_{n}^{k}(y)|y|^{2 k} d y \\
& =\int_{\mathbb{R}} \sum_{n=0}^{+\infty} e^{-t(2 n+2 k+1)} h_{n}^{k}(x) h_{n}^{k}(y) f(y)|y|^{2 k} d y=\int_{\mathbb{R}} P_{k}(t, x, y) f(y)|y|^{2 k} d y .
\end{aligned}
$$

Interchanging the order of summation and integration is justified by Lebesgue's dominated convergence theorem since

$$
\begin{aligned}
& \sum_{n=0}^{+\infty} e^{-t(2 n+2 k+1)} \int_{\mathbb{R}}\left|h_{n}^{k}(x) h_{n}^{k}(y) f(y) \| y\right|^{2 k} d y \\
& \quad \leq \sum_{n=0}^{+\infty} e^{-t(2 n+2 k+1)}\left\|h_{n}^{k}\right\|_{\infty}\left\|h_{n}^{k}\right\|_{k, p^{\prime}}\|f\|_{k, p}<+\infty
\end{aligned}
$$


Mehler's formula established by Margit Rösler for Dunkl-Hermite polynomials (see [8]), adapted to Dunkl-Hermite functions $\left\{h_{n}^{k}\right\}_{n \geq 0}$ reads

$$
\sum_{n=0}^{+\infty} r^{n} h_{n}^{k}(y) h_{n}^{k}(z)=\frac{c_{k}}{\left(1-r^{2}\right)^{k+\frac{1}{2}}} e^{-\frac{1}{2}\left(\frac{1+r^{2}}{1-r^{2}}\right)\left(y^{2}+z^{2}\right)} E_{k}\left(\frac{2 r y}{1-r^{2}}, z\right), \quad 0<r<1,
$$

where $c_{k}$ is the constant defined by

$$
c_{k}=\left(\int_{\mathbb{R}} e^{-x^{2}}|x|^{2 k} d x\right)^{-1}
$$

and $E_{k}$ is the Dunkl kernel expressed in terms of the normalized Bessel function

$$
E_{k}(x, y)=j_{k-\frac{1}{2}}(i x y)+\frac{x y}{2 k+1} j_{k+\frac{1}{2}}(i x y),
$$

where

$$
j_{\alpha}(z)=\Gamma(\alpha+1) \sum_{n=0}^{+\infty} \frac{(-1)^{n}\left(\frac{z}{2}\right)^{2 n}}{n ! \Gamma(n+\alpha+1)}, \quad \alpha \geq-\frac{1}{2} .
$$

Set

$$
U_{k}(r, y, z):=\sum_{n=0}^{+\infty} r^{n} h_{n}^{k}(y) h_{n}^{k}(z), \quad 0<r<1 .
$$

Proposition 5. The kernel $U_{k}$ satisfies the following properties

(i) $U_{k}(r, y, z) \geq 0$,

(ii) $U_{k}(r, y, z)=U_{k}(r, z, y)$,

(iii) $\quad\left\|U_{k}(r, y, \cdot)\right\|_{k, 1}=\left(\frac{2}{1+r^{2}}\right)^{k+\frac{1}{2}} e^{-\frac{1}{2}\left(\frac{1-r^{2}}{1+r^{2}}\right) y^{2}}$.

Proof. (i) and (ii) are obvious, let us therefore prove (iii).

$$
\begin{aligned}
\left\|U_{k}(r, y, \cdot)\right\|_{k, 1} & =\frac{c_{k}}{\left(1-r^{2}\right)^{k+\frac{1}{2}}} \int_{\mathbb{R}} e^{-\frac{1}{2}\left(\frac{1+r^{2}}{1-r^{2}}\right)\left(y^{2}+z^{2}\right)} E_{k}\left(\frac{2 r y}{1-r^{2}}, z\right)|z|^{2 k} d z \\
& =\frac{c_{k}}{\left(1-r^{2}\right)^{k+\frac{1}{2}}} e^{-\frac{1}{2}\left(\frac{1+r^{2}}{1-r^{2}}\right) y^{2}} \int_{\mathbb{R}} e^{-\frac{1}{2}\left(\frac{1+r^{2}}{1-r^{2}}\right) z^{2}} E_{k}\left(\frac{2 r y}{1-r^{2}}, z\right)|z|^{2 k} d z .
\end{aligned}
$$

Performing the change of variables $u=\sqrt{\frac{1+r^{2}}{1-r^{2}}} z$, and using the following identity (see [2])

$$
\int_{\mathbb{R}} E_{k}(x, y) e^{-\frac{x^{2}}{2}}|x|^{2 k} d x=2^{k+\frac{1}{2}} c_{k}^{-1} e^{\frac{y^{2}}{2}}
$$

we are done.

Proposition 6. The heat-diffusion integral $G_{k}(f)$ is a $C^{\infty}$ function on $\mathbb{R}_{+} \times \mathbb{R}$ satisfying the differential-difference equation

$$
\left(L_{k, x}-\frac{\partial}{\partial t}\right) G_{k}(f)(t, x)=0
$$

(here $L_{k, x}$ means that the operator $L_{k}$ acts on the variable $x$ ). 
Proof. On the one hand, one has for all $m \in \mathbb{N}$

$$
\frac{\partial^{m}}{\partial t^{m}} G_{k}(f)(t, x)=\sum_{n=0}^{+\infty}(-1)^{m}(2 n+2 k+1)^{m} e^{-t(2 n+2 k+1)} a_{n}^{k}(f) h_{n}^{k}(x) .
$$

On the other hand, it is easy to see that

$$
\left(h_{n}^{k}\right)^{\prime}(x)=e^{-\frac{x^{2}}{2}} \frac{\partial}{\partial x} H_{n}^{k}(x)-x h_{n}^{k}(x),
$$

thus for fixed $t$, the series (5) can be differentiated termwisely with respect to $x$. A similar argument holds for higher derivatives and then $G_{k}(f)$ is $C^{\infty}$ on $\mathbb{R}_{+} \times \mathbb{R}$. Differentiating term by term shows that $G_{k}(f)(t, x)$ satisfies (4).

Theorem 1. The heat-diffusion integral $G_{k}(f)(t, \cdot), t>0$, satisfies

$$
\left\|G_{k}(f)(t, \cdot)\right\|_{k, p} \leq(\cosh (2 t))^{-\left(k+\frac{1}{2}\right)}\|f\|_{k, p} .
$$

Proof. Using

$$
P_{k}(t, x, y)=e^{-t(2 k+1)} U_{k}\left(e^{-2 t}, x, y\right)
$$

and (3), we obtain

$$
\int_{\mathbb{R}} P_{k}(t, x, y)|y|^{2 k} d y=(\cosh (2 t))^{-\left(k+\frac{1}{2}\right)} e^{-\frac{1}{2} \tanh (2 t) x^{2}} .
$$

By Hölder's inequality, it follows that

$$
\left|G_{k}(f)(t, x)\right|^{p} \leq(\cosh (2 t))^{-\frac{p\left(k+\frac{1}{2}\right)}{p^{\prime}}} \int_{\mathbb{R}}|f(y)|^{p}\left|P_{k}(t, x, y)\right||y|^{2 k} d y,
$$

where $p^{\prime}$ is the conjugate exponent of $p$. Integration with respect to $x$ and using Fubini's Theorem yield

$$
\left\|G_{k}(f)(t, \cdot)\right\|_{k, p} \leq(\cosh (2 t))^{-\left(k+\frac{1}{2}\right)}\|f\|_{k, p} .
$$

\subsection{Poisson integral}

In this subsection, we introduce the Poisson integral and we give its $L^{p}$ boundedness.

Definition 2. The Poisson integral $F_{k}(f)$ of $f$ is defined by

$$
F_{k}(f)(t, x)=\sum_{n=0}^{+\infty} e^{-t \sqrt{2 n+2 k+1}} a_{n}^{k}(f) h_{n}^{k}(x), \quad t>0, \quad x \in \mathbb{R} .
$$

Again the defining series is convergent by Propositions 1 and 2 .

Proposition 7. $F_{k}(f)$ possesses the following integral representation

$$
F_{k}(f)(t, x)=\int_{\mathbb{R}} f(y) A_{k}(t, x, y)|y|^{2 k} d y,
$$

where

$$
A_{k}(t, x, y)=\frac{t}{\sqrt{4 \pi}} \int_{0}^{+\infty} P_{k}(u, x, y) u^{-\frac{3}{2}} e^{-\frac{t^{2}}{4 u}} d u .
$$

$A_{k}$ is called the Poisson kernel associated with $\left\{h_{n}^{k}\right\}_{n \geq 0}$. 
Proof. By using the subordination formula

$$
e^{-\beta}=\frac{\beta}{\sqrt{4 \pi}} \int_{0}^{+\infty} e^{-s} s^{-\frac{3}{2}} e^{-\frac{\beta^{2}}{4 s}} d s, \quad \beta>0,
$$

we obtain an integral form of $F_{k}(f)(t, x)$ by writing

$$
\begin{aligned}
F_{k}(f)(t, x) & =\sum_{n=0}^{+\infty} \frac{t \sqrt{2 n+2 k+1}}{\sqrt{4 \pi}} h_{n}^{k}(x) \int_{0}^{+\infty} e^{-s} s^{-\frac{3}{2}} e^{-\frac{t^{2}(2 n+2 k+1)}{4 s}} d s \int_{\mathbb{R}} f(y) h_{n}^{k}(y)|y|^{2 k} d y \\
& =\frac{t}{\sqrt{4 \pi}} \sum_{n=0}^{+\infty} h_{n}^{k}(x) \int_{0}^{+\infty} u^{-\frac{3}{2}} e^{-u(2 n+2 k+1)} e^{-\frac{t^{2}}{4 u}} d u \int_{\mathbb{R}} f(y) h_{n}^{k}(y)|y|^{2 k} d y \\
& =\frac{t}{\sqrt{4 \pi}} \int_{\mathbb{R}} f(y) \int_{0}^{+\infty} \sum_{n=0}^{+\infty} e^{-(2 n+2 k+1) u} h_{n}^{k}(x) h_{n}^{k}(y) u^{-\frac{3}{2}} e^{-\frac{t^{2}}{4 u}} d u|y|^{2 k} d y \\
& =\frac{t}{\sqrt{4 \pi}} \int_{\mathbb{R}} f(y) \int_{0}^{+\infty} P_{k}(u, x, y) u^{-\frac{3}{2}} e^{-\frac{t^{2}}{4 u}} d u|y|^{2 k} d y=\int_{\mathbb{R}} f(y) A_{k}(t, x, y)|y|^{2 k} d y .
\end{aligned}
$$

The same argument used for the heat-diffusion integral implies that $F_{k}(f)$ is $C^{\infty}$ on $\mathbb{R}_{+} \times \mathbb{R}$ and satisfies

$$
\left(L_{k, x}+\frac{\partial^{2}}{\partial t^{2}}\right) F_{k}(f)(t, x)=0 .
$$

Theorem 2. $F_{k}(f)(t, \cdot) \in L^{p}\left(\mathbb{R},|x|^{2 k} d x\right)$ and

$$
\left\|F_{k}(f)(t, \cdot)\right\|_{k, p} \leq 2^{k+\frac{1}{2}} e^{-t \sqrt{2 k+1}}\|f\|_{k, p} .
$$

Proof. One has

$$
\begin{aligned}
F_{k}(f)(t, x) & =\int_{\mathbb{R}} A_{k}(t, x, y) f(y)|y|^{2 k} d y=\frac{t}{\sqrt{4 \pi}} \int_{\mathbb{R}} \int_{0}^{+\infty} P_{k}(u, x, y) u^{-\frac{3}{2}} e^{-\frac{t^{2}}{4 u}} d u f(y)|y|^{2 k} d y \\
& =\frac{t}{\sqrt{4 \pi}} \int_{0}^{+\infty}\left(\int_{\mathbb{R}} P_{k}(u, x, y) f(y)|y|^{2 k} d y\right) u^{-\frac{3}{2}} e^{-\frac{t^{2}}{4 u}} d u \\
& =\frac{t}{\sqrt{4 \pi}} \int_{0}^{+\infty} G_{k}(f)(u, x) u^{-\frac{3}{2}} e^{-\frac{t^{2}}{4 u}} d u .
\end{aligned}
$$

It follows that

$$
\left\|F_{k}(f)(t, \cdot)\right\|_{k, p} \leq \frac{t}{\sqrt{4 \pi}} \int_{0}^{+\infty}\left\|G_{k}(f)(u, \cdot)\right\|_{k, p} u^{-\frac{3}{2}} e^{-\frac{t^{2}}{4 u}} d u \leq 2^{k+\frac{1}{2}} e^{-t \sqrt{2 k+1}}\|f\|_{k, p} .
$$

\section{Hilbert transforms}

The operator $\left(-L_{k}\right)$ is positive and symmetric in $L^{2}\left(\mathbb{R},|x|^{2 k} d x\right)$ on the domain $C_{c}^{\infty}(\mathbb{R})$. It may be easily checked that the operator $\left(-\mathcal{L}_{k}\right)$ given by

$$
\left(-\mathcal{L}_{k}\right)\left(\sum_{n=0}^{+\infty} a_{n}^{k}(f) h_{n}^{k}\right)=\sum_{n=0}^{+\infty}(2 n+2 k+1) a_{n}^{k}(f) h_{n}^{k}
$$

on the domain

$$
\operatorname{Dom}\left(-\mathcal{L}_{k}\right)=\left\{f \in L^{2}\left(\mathbb{R},|x|^{2 k} d x\right): \sum_{n=0}^{+\infty}\left|(2 n+2 k+1) a_{n}^{k}(f)\right|^{2}<+\infty\right\}
$$


is a self-adjoint extension of $\left(-L_{k}\right)$, has the spectrum $\{2 n+2 k+1\}$ and admits the spectral decomposition

$$
\left(-\mathcal{L}_{k}\right) f=\sum_{n=0}^{+\infty}(2 n+2 k+1) a_{n}^{k}(f) h_{n}^{k}, \quad f \in \operatorname{Dom}\left(-\mathcal{L}_{k}\right) .
$$

Following [10, p. 57] the Hilbert transforms associated with the Dunkl-Hermite operator are formally given by

$$
\mathcal{H}_{k}^{ \pm}=\left(T_{k} \pm x\right)\left(-L_{k}\right)^{-\frac{1}{2}}
$$

Note, that if $f \sim \sum_{n=0}^{+\infty} a_{n}^{k}(f) h_{n}^{k}$, then again formally,

$$
\mathcal{H}_{k}^{+} f \sim \sum_{n=0}^{+\infty} a_{n}^{k}(f) \frac{\theta(n, k)}{\sqrt{2 n+2 k+1}} h_{n-1}^{k}, \quad \mathcal{H}_{k}^{-} f \sim-\sum_{n=0}^{+\infty} a_{n}^{k}(f) \frac{\theta(n+1, k)}{\sqrt{2 n+2 k+1}} h_{n+1}^{k},
$$

where

$$
\theta(n, k)= \begin{cases}\sqrt{2 n} & \text { if } n \text { is even } \\ \sqrt{2 n+4 k} & \text { if } n \text { is odd. }\end{cases}
$$

We use the convention that $h_{n-1}^{k}=0$ if $n=0$. It is clear that $\mathcal{H}_{k}^{ \pm}$are defined on $L^{2}\left(\mathbb{R},|x|^{2 k} d x\right)$ by

$$
\mathcal{H}_{k}^{+} f=\sum_{n=0}^{+\infty} a_{n}^{k}(f) \frac{\theta(n, k)}{\sqrt{2 n+2 k+1}} h_{n-1}^{k}, \quad \mathcal{H}_{k}^{-} f=-\sum_{n=0}^{+\infty} a_{n}^{k}(f) \frac{\theta(n+1, k)}{\sqrt{2 n+2 k+1}} h_{n+1}^{k} .
$$

To proceed to a deeper analysis of these definitions, in particular to consider $\mathcal{H}_{k}^{ \pm}$on a wider class of functions, we define the kernels $R_{k}^{ \pm}(x, y)$ by

$$
R_{k}^{ \pm}(x, y)=\frac{1}{\sqrt{\pi}}\left(T_{k, x} \pm x\right) \int_{0}^{+\infty} P_{k}(t, x, y) t^{-\frac{1}{2}} d t=\frac{1}{\sqrt{\pi}} \int_{0}^{+\infty}\left(T_{k, x} \pm x\right) P_{k}(t, x, y) t^{-\frac{1}{2}} d t
$$

It will be shown in Proposition 8 that the second integral in (8) converges.

We have

$$
\begin{aligned}
P_{k}(t, x, y) & =\sum_{n=0}^{+\infty} e^{-t(2 n+2 k+1)} h_{n}^{k}(x) h_{n}^{k}(y) \\
& =\frac{c_{k}}{2^{k+\frac{1}{2}}(\sinh (2 t))^{k+\frac{1}{2}}} e^{-\frac{1}{2} \operatorname{coth}(2 t)\left(x^{2}+y^{2}\right)} E_{k}\left(\frac{x}{\sinh (2 t)}, y\right) .
\end{aligned}
$$

The change of variables $2 t=\log \left(\frac{1+s}{1-s}\right)$ furnishes a useful variant of (8):

$$
R_{k}^{ \pm}(x, y)=\frac{\sqrt{2}}{\sqrt{\pi}} \int_{0}^{1}\left(T_{k, x} \pm x\right) K_{s}(x, y)\left(\log \left(\frac{1+s}{1-s}\right)\right)^{-\frac{1}{2}} \frac{d s}{1-s^{2}}
$$

where

$$
K_{s}(x, y)=c_{k}\left(\frac{1-s^{2}}{4 s}\right)^{k+\frac{1}{2}} e^{-\frac{1}{4}\left(s+\frac{1}{s}\right)\left(x^{2}+y^{2}\right)} E_{k}\left(\left(\frac{1-s^{2}}{2 s}\right) x, y\right) .
$$


We then write

$$
R_{k}^{ \pm}(x, y)=\frac{\sqrt{2}}{\sqrt{\pi}}\left[R_{k, 1}(x, y) \pm R_{k, 2}(x, y)\right]
$$

where

$$
R_{k, 1}(x, y)=\int_{0}^{1} T_{k, x} K_{s}(x, y)\left(\log \left(\frac{1+s}{1-s}\right)\right)^{-\frac{1}{2}} \frac{d s}{1-s^{2}}
$$

and

$$
R_{k, 2}(x, y)=\int_{0}^{1} x K_{s}(x, y)\left(\log \left(\frac{1+s}{1-s}\right)\right)^{-\frac{1}{2}} \frac{d s}{1-s^{2}} .
$$

Proposition 8. There exists a positive constant $C$ such that for $(x, y) \in \Delta^{c}=\left\{(x, y) \in \mathbb{R}^{2}\right.$ : $x \neq y\}$, the kernels $R_{k, 1}(x, y)$ and $R_{k, 2}(x, y)$ satisfy

$$
\begin{aligned}
& \left|R_{k, 1}(x, y)\right| \leq \frac{C}{|x-y|}, \\
& \left|R_{k, 2}(x, y)\right| \leq \frac{C}{|x-y|} .
\end{aligned}
$$

Proof. We start with proving (10). We have

$$
\left|R_{k, 2}(x, y)\right| \leq C \int_{0}^{1} \beta(s)|x| e^{-\frac{1}{4}\left(s+\frac{1}{s}\right)\left(x^{2}+y^{2}\right)} E_{k}\left(\left(\frac{1-s^{2}}{2 s}\right) x, y\right) d s
$$

where we let

$$
\beta(s)=(1-s)^{k-\frac{1}{2}} s^{-\left(k+\frac{1}{2}\right)}\left(\log \left(\frac{1+s}{1-s}\right)\right)^{-\frac{1}{2}} .
$$

Using the following estimate (see [9])

$$
E_{k}\left(\left(\frac{1-s^{2}}{2 s}\right) x, y\right) \leq e^{\left(\frac{1-s^{2}}{2 s}\right)|x y|},
$$

the same reasoning as in [11, pp. 460-461] in the classical case gives the result. In order to estimate $R_{k, 1}$, write

$$
T_{k, x} K_{s}(x, y)=-\frac{1}{2}\left[s(x+y)+\frac{1}{s}(x-y)\right] K_{s}(x, y)
$$

to see that

$$
\left|R_{k, 1}(x, y)\right| \leq C \int_{0}^{1} \beta(s)\left[s|x+y|+\frac{1}{s}|x-y|\right] e^{-\frac{1}{4}\left(s+\frac{1}{s}\right)\left(x^{2}+y^{2}\right)} E_{k}\left(\left(\frac{1-s^{2}}{2 s}\right) x, y\right) d s,
$$

and use the same above arguments used to get the bound for $R_{k, 1}$.

Proposition 9. There exists a positive constant $C$ such that for $(x, y) \in \Delta^{c}=\left\{(x, y) \in \mathbb{R}^{2}\right.$ : $x \neq y\}$, if $|x-y| \geq 2\left|x-x^{\prime}\right|$, then

$$
\begin{aligned}
& \left|R_{k, 1}(x, y)-R_{k, 1}\left(x^{\prime}, y\right)\right| \leq \frac{C\left|x-x^{\prime}\right|}{|x-y|^{2}}, \\
& \left|R_{k, 2}(x, y)-R_{k, 2}\left(x^{\prime}, y\right)\right| \leq \frac{C\left|x-x^{\prime}\right|}{|x-y|^{2}} .
\end{aligned}
$$


Proof. Start with

$$
\begin{aligned}
\left|R_{k, 2}(x, y)-R_{k, 2}\left(x^{\prime}, y\right)\right| \leq & \int_{0}^{1}\left|x K_{s}(x, y)-x^{\prime} K_{s}\left(x^{\prime}, y\right)\right|\left(\log \left(\frac{1+s}{1-s}\right)\right)^{-\frac{1}{2}} \frac{d s}{1-s^{2}} \\
\leq & C \int_{0}^{1} \beta(s) \mid x e^{-\frac{1}{4}\left(s+\frac{1}{s}\right)\left(x^{2}+y^{2}\right)} E_{k}\left(\left(\frac{1-s^{2}}{2 s}\right) x, y\right) \\
& -x^{\prime} e^{-\frac{1}{4}\left(s+\frac{1}{s}\right)\left(x^{\prime 2}+y^{2}\right)} E_{k}\left(\left(\frac{1-s^{2}}{2 s}\right) x^{\prime}, y\right) \mid d s .
\end{aligned}
$$

Using the following estimates (see [9])

$$
\begin{aligned}
& \left|\frac{\partial}{\partial x}\left(E_{k}\left(\left(\frac{1-s^{2}}{2 s}\right) x, y\right)\right)\right| \leq\left(\frac{1-s^{2}}{2 s}\right)|y| E_{k}\left(\left(\frac{1-s^{2}}{2 s}\right) x, y\right), \\
& E_{k}\left(\left(\frac{1-s^{2}}{2 s}\right) x, y\right) \leq e^{\left(\frac{1-s^{2}}{2 s}\right)|x y|},
\end{aligned}
$$

then the same reasoning as in [11, pp. 461-463] in the classical case gives the result.

Considering $R_{k, 1}$, we have

$$
\begin{aligned}
\left|R_{k, 1}(x, y)-R_{k, 1}\left(x^{\prime}, y\right)\right| \leq \int_{0}^{1}\left|T_{k, x} K_{s}(x, y)-T_{k, x} K_{s}\left(x^{\prime}, y\right)\right|\left(\log \left(\frac{1+s}{1-s}\right)\right)^{-\frac{1}{2}} \frac{d s}{1-s^{2}} \\
\leq C \int_{0}^{1} \beta(s) \mid\left[s(x+y)+\frac{1}{s}(x-y)\right] e^{-\frac{1}{4}\left(s+\frac{1}{s}\right)\left(x^{2}+y^{2}\right)} E_{k}\left(\left(\frac{1-s^{2}}{2 s}\right) x, y\right) \\
-\left[s\left(x^{\prime}+y\right)+\frac{1}{s}\left(x^{\prime}-y\right)\right] e^{-\frac{1}{4}\left(s+\frac{1}{s}\right)\left(x^{\prime 2}+y^{2}\right)} E_{k}\left(\left(\frac{1-s^{2}}{2 s}\right) x^{\prime}, y\right) \mid d s .
\end{aligned}
$$

The proof of (11) follows the same steps of the one of (12).

Proposition 10. There exists a positive constant $C$ such that for $(x, y) \in \Delta^{c}=\left\{(x, y) \in \mathbb{R}^{2}\right.$ : $x \neq y\}$, if $|x-y| \geq 2\left|y-y^{\prime}\right|$ then

$$
\begin{aligned}
& \left|R_{k, 2}(x, y)-R_{k, 2}\left(x, y^{\prime}\right)\right| \leq \frac{C\left|y-y^{\prime}\right|}{|x-y|^{2}}, \\
& \left|R_{k, 1}(x, y)-R_{k, 1}\left(x, y^{\prime}\right)\right| \leq \frac{C\left|y-y^{\prime}\right|}{|x-y|^{2}} .
\end{aligned}
$$

Proof. The proofs of (13) and (14) are quite similar to the ones of (11) and (12).

Lemma 1. Given $m, m=1,2, \ldots$, and $f \in C_{c}^{\infty}(\mathbb{R})$ there exists $C=C_{m, f}>0$ such that

$$
\left|\left\langle f, h_{n}^{k}\right\rangle\right| \leq C(2 n+2 k+1)^{-m},
$$

where

$$
\langle f, g\rangle=\int_{\mathbb{R}} f(x) \overline{g(x)}|x|^{2 k} d x .
$$

\section{Proof.}

$$
\left|\left\langle f, h_{n}^{k}\right\rangle\right|=\left.\left|\int_{\mathbb{R}} f(t) h_{n}^{k}(t)\right| t\right|^{2 k} d t|=|(-(2 n+2 k+1))^{-m}\left\langle L_{k}^{m} f, h_{n}^{k}\right\rangle \mid \leq C(2 n+2 k+1)^{-m} .
$$


Theorem 3. Let $f, g \in C_{c}^{\infty}(\mathbb{R})$ with disjoint supports. Then

$$
\left\langle\mathcal{H}_{k}^{ \pm} f, g\right\rangle=\int_{\mathbb{R}} \int_{\mathbb{R}} R_{k}^{ \pm}(x, y) f(y) \overline{g(x)}|y|^{2 k} d y|x|^{2 k} d x
$$

Proof. We first consider $\mathcal{H}_{k}^{+}$. Let

$$
f=\sum_{n=0}^{+\infty} a_{n}^{k}(f) h_{n}^{k} \quad \text { and } \quad g=\sum_{n=0}^{+\infty} b_{n}^{k}(g) h_{n}^{k}
$$

Then

$$
\mathcal{H}_{k}^{+} f=\sum_{n=0}^{+\infty} \frac{\theta(n, k)}{\sqrt{2 n+2 k+1}} a_{n}^{k}(f) h_{n-1}^{k} .
$$

The convergence of the three series is in $\left.L^{2}\left(\mathbb{R},|x|^{2 k} d x\right)\right)$ and by Parseval's identity

$$
\left\langle\mathcal{H}_{k}^{+} f, g\right\rangle=\sum_{n=0}^{+\infty} \frac{\theta(n, k)}{\sqrt{2 n+2 k+1}} a_{n}^{k}(f) \overline{b_{n-1}^{k}(g)} \text {. }
$$

We will show that the right sides in (15) and (16) coincide. Note that we can see as in Proposition 8 that

$$
\int_{0}^{+\infty}\left|\left(T_{k, x}+x\right) P_{k}(t, x, y)\right| t^{-\frac{1}{2}} d t \leq \frac{C}{|x-y|} .
$$

This result and the assumption made on the supports of $f$ and $g$ show that

$$
\int_{\mathbb{R}} \int_{\mathbb{R}} \int_{0}^{+\infty}\left|\left(T_{k, x}+x\right) P_{k}(t, x, y)\right| t^{-\frac{1}{2}} d t|\overline{g(x)} f(y)||y|^{2 k} d y|x|^{2 k} d x<+\infty .
$$

Now,

$$
\begin{aligned}
& \int_{\mathbb{R}} \int_{\mathbb{R}} R_{k}^{+}(x, y) f(y) \overline{g(x)}|y|^{2 k} d y|x|^{2 k} d x \\
& =\frac{1}{\sqrt{\pi}} \int_{\mathbb{R}} \int_{\mathbb{R}} \int_{0}^{+\infty}\left\{\left(T_{k, x}+x\right) P_{k}(t, x, y)\right\} t^{-\frac{1}{2}} d t f(y) \overline{g(x)}|y|^{2 k} d y|x|^{2 k} d x \\
& =\frac{1}{\sqrt{\pi}} \int_{0}^{+\infty} \int_{\mathbb{R}} \int_{\mathbb{R}}\left\{\left(T_{k, x}+x\right)\left(\sum_{n=0}^{+\infty} e^{-t(2 n+2 k+1)} h_{n}^{k}(x) h_{n}^{k}(y)\right)\right\} \overline{g(x)} f(y)|x|^{2 k} d x|y|^{2 k} d y t^{-\frac{1}{2}} d t \\
& =\frac{1}{\sqrt{\pi}} \int_{0}^{+\infty} \int_{\mathbb{R}} \int_{\mathbb{R}}\left\{\sum_{n=0}^{+\infty} e^{-t(2 n+2 k+1)} \theta(n, k) h_{n-1}^{k}(x) h_{n}^{k}(y)\right\} \overline{g(x)} f(y)|x|^{2 k} d x|y|^{2 k} d y t^{-\frac{1}{2}} d t \\
& =\frac{1}{\sqrt{\pi}} \int_{0}^{+\infty} \int_{\mathbb{R}}\left\{\sum_{n=0}^{+\infty} e^{-t(2 n+2 k+1)} \theta(n, k) \overline{b_{n-1}^{k}(g)} h_{n}^{k}(y)\right\} f(y)|y|^{2 k} d y t^{-\frac{1}{2}} d t \\
& =\frac{1}{\sqrt{\pi}} \int_{0}^{+\infty}\left\{\sum_{n=0}^{+\infty} e^{-t(2 n+2 k+1)} \theta(n, k) a_{n}^{k}(f) \overline{b_{n-1}^{k}(g)}\right\} t^{-\frac{1}{2}} d t=\left\langle\mathcal{H}_{k}^{+} f, g\right\rangle .
\end{aligned}
$$

Note that Fubini's theorem is justified by (17). Recall that

$$
\mathcal{H}_{k}^{-} f=-\sum_{n=0}^{+\infty} \frac{\theta(n+1, k)}{\sqrt{2 n+2 k+1}} a_{n}^{k}(f) h_{n+1}^{k},
$$


then one gets similarly

$$
\begin{aligned}
\int_{\mathbb{R}} \int_{\mathbb{R}} & R_{k}^{-}(x, y) f(y) \overline{g(x)}|y|^{2 k} d y|x|^{2 k} d x \\
& =\frac{1}{\sqrt{\pi}} \int_{\mathbb{R}} \int_{\mathbb{R}} \int_{0}^{+\infty}\left\{\left(T_{k, x}-x\right) P_{k}(t, x, y)\right\} t^{-\frac{1}{2}} d t f(y) \overline{g(x)}|y|^{2 k} d y|x|^{2 k} d x \\
& =-\sum_{n=0}^{+\infty} \frac{\theta(n+1, k)}{\sqrt{2 n+2 k+1}} a_{n}^{k}(f) \overline{b_{n+1}^{k}(g)}=\left\langle\mathcal{H}_{k}^{-} f, g\right\rangle .
\end{aligned}
$$

Theorem 4. For almost every $x$ in $\mathbb{R}$, the Hilbert transforms are given by

$$
\mathcal{H}_{k}^{ \pm} f(x)=\lim _{\epsilon \longrightarrow 0} \int_{|x-y|>\epsilon} f(y) R_{k}^{ \pm}(x, y)|y|^{2 k} d y .
$$

Proof. We have

$$
\int_{|x-y|>\epsilon} f(y) R_{k}^{ \pm}(x, y)|y|^{2 k} d y=\int_{|x-y|>\epsilon} f(y)|y|^{\frac{2 k}{p}}|y|^{\frac{2 k}{p^{\prime}}} R_{k}^{ \pm}(x, y) d y=\int_{|x-y|>\epsilon} g(y) W_{k}^{ \pm}(x, y) d y,
$$

where $p^{\prime}$ is the conjugate exponent of $p$,

$$
g(y)=f(y)|y|^{\frac{2 k}{p}}, \quad g \in L^{p}(\mathbb{R}, d x), \quad W_{k}^{ \pm}(x, y)=|y|^{\frac{2 k}{p^{\prime}}} R_{k}^{ \pm}(x, y),
$$

$W_{k}^{ \pm}(x, y)$ are Calderón-Zygmund kernels (see Propositions 8, 9 and 10). It follows that

$$
\lim _{\epsilon \longrightarrow 0} \int_{|x-y|>\epsilon} f(y) R_{k}^{ \pm}(x, y)|y|^{2 k} d y=\lim _{\epsilon \longrightarrow 0} \int_{|x-y|>\epsilon} g(y) W_{k}^{ \pm}(x, y) d y
$$

exist for almost every $x$ (see $[5$, p. 55]).

Remark 1. For $f \in L^{2}\left(\mathbb{R},|x|^{2 k} d x\right)$, we have

$$
\mathcal{F}_{k}\left(\mathcal{H}_{k}^{ \pm} f\right)(x)= \pm i \mathcal{H}_{k}^{ \pm}\left(\mathcal{F}_{k} f\right)(x)
$$

where $\mathcal{F}_{k}$ is the Plancherel Dunkl transform, (see [1]).

Theorem 5. The operators $\mathcal{H}_{k}^{ \pm}$are bounded on $L^{p}\left(\mathbb{R},|x|^{2 k} d x\right), 1<p<+\infty$.

Proof. Consider the truncated operators

$$
\begin{aligned}
\mathcal{H}_{\epsilon, k}^{ \pm} f(x) & =\int_{|x-y|>\epsilon} f(y) R_{k}^{ \pm}(x, y)|y|^{2 k} d y \\
\left\|\mathcal{H}_{k}^{ \pm} f\right\|_{k, p}^{p} & =\int_{\mathbb{R}}\left|\mathcal{H}_{k}^{ \pm} f(x)\right|^{p}|x|^{2 k} d x=\int_{\mathbb{R}}\left|\lim _{\epsilon \longrightarrow 0} \mathcal{H}_{\epsilon, k}^{ \pm} f(x)\right|^{p}|x|^{2 k} d x \\
& =\int_{\mathbb{R}} \lim _{\epsilon \longrightarrow 0}\left|\mathcal{H}_{\epsilon, k}^{ \pm} f(x)\right|^{p}|x|^{2 k} d x=\int_{R} \liminf _{\epsilon \longrightarrow 0}\left|\mathcal{H}_{\epsilon, k}^{ \pm} f(x)\right|^{p}|x|^{2 k} d x \\
& \leq \liminf _{\epsilon \longrightarrow 0} \int_{\mathbb{R}}\left|\mathcal{H}_{\epsilon, k}^{ \pm} f(x)\right|^{p}|x|^{2 k} d x=\left.\left.\liminf _{\epsilon \longrightarrow 0} \int_{\mathbb{R}}|| x\right|^{\frac{2 k}{p}} \mathcal{H}_{\epsilon, k}^{ \pm} f(x)\right|^{p} d x
\end{aligned}
$$

Yet,

$$
|x|^{\frac{2 k}{p}} \mathcal{H}_{\epsilon, k}^{ \pm} f(x)=\int_{|x-y|>\epsilon} f(y)|x|^{\frac{2 k}{p}} R_{k}^{ \pm}(x, y)|y|^{2 k} d y=\int_{|x-y|>\epsilon} g(y) Z_{k}^{ \pm}(x, y) d y,
$$


where

$$
g(y)=f(y)|y|^{\frac{2 k}{p}}, g \in L^{p}(\mathbb{R}, d x), \quad Z_{k}^{ \pm}(x, y)=|x|^{\frac{2 k}{p}}|y|^{\frac{2 k}{p^{\prime}}} R_{k}^{ \pm}(x, y) .
$$

$Z_{k}^{ \pm}(x, y)$ are Calderón-Zygmund kernels (see Propositions 8, 9 and 10). Let

$$
S_{g}^{ \pm}(x)=\int_{\mathbb{R}} g(y) Z_{k}^{ \pm}(x, y) d y
$$

The operators $S_{g}^{ \pm}$are Calderón-Zygmund type associated with the Calderón-Zygmund kernels $Z_{k}^{ \pm}(x, y)($ see $[5$, p. 48$])$, then

$$
\sup _{\epsilon>0}\left|\int_{|x-y|>\epsilon} g(y) Z_{k}^{ \pm}(x, y) d y\right|
$$

are bounded on $L^{p}(\mathbb{R}, d x)$ for $1<p<+\infty$ (see [5, p. 56]). Consequently, there exists a positive constant $C=C_{p}$ such that if $f \in L^{p}\left(\mathbb{R},|x|^{2 k} d x\right)$ then

$$
\left\|\mathcal{H}_{k}^{ \pm} f\right\|_{k, p} \leq C\|f\|_{k, p}
$$

Lemma 2. There exists a positive constant $C$ such that for $f \in L^{1}\left(\mathbb{R},|x|^{2 k} d x\right), \lambda>0$, we have

$$
\int_{\left\{x \in \mathbb{R}: \sup _{\epsilon>0}\left|\mathcal{H}_{\epsilon, k}^{ \pm} f(x)\right|>\lambda\right\}} d x \leq \frac{C}{\lambda}\|f\|_{k, 1}
$$

Proof. We have

$$
\mathcal{H}_{\epsilon, k}^{ \pm} f(x)=\int_{|x-y|>\epsilon} f(y) R_{k}^{ \pm}(x, y)|y|^{2 k} d y=\int_{|x-y|>\epsilon} g(y) W_{k}^{ \pm}(x, y) d y
$$

where

$$
g(y)=f(y)|y|^{\frac{2 k}{p}} \quad \text { and } \quad W_{k}^{ \pm}(x, y)=|y|^{\frac{2 k}{p^{\prime}}} R_{k}^{ \pm}(x, y) .
$$

Let

$$
S_{g}^{ \pm}(x)=\int_{\mathbb{R}} g(y) W_{k}^{ \pm}(x, y) d y
$$

The operators $S_{g}^{ \pm}$are Calderón-Zygmund operators associated with the Calderón-Zygmund kernels $W_{k}^{ \pm}(x, y)$ then there exists a positive constant $C$ such that for $\lambda>0$ and $f \in L^{1}\left(\mathbb{R},|x|^{2 k} d x\right)$, we have

$$
\int_{\left\{x \in \mathbb{R}: \sup _{\epsilon>0}\left|\mathcal{H}_{\epsilon, k}^{ \pm} f(x)\right|>\lambda\right\}} d x \leq \frac{C}{\lambda}\|f\|_{k, 1} .
$$

As a by-product, we have the following.

Theorem 6. There exists a positive constant $C$ such that for $f \in L^{1}\left(\mathbb{R},|x|^{2 k} d x\right)$, we have

$$
\sup _{\lambda>0}\left(\lambda \int_{\left\{x \in \mathbb{R}:\left|\mathcal{H}_{k}^{ \pm} f(x)\right|>\lambda\right\}} d x\right) \leq C\|f\|_{k, 1} .
$$




\section{Conjugate Poisson integrals}

Dunkl-Hermite functions allow to define the conjugate Poisson integrals.

Definition 3. The conjugate Poisson integrals $f_{k}^{ \pm}(t, x)$ of $f$ are defined by

$$
\begin{aligned}
& f_{k}^{+}(t, x)=\sum_{n=0}^{+\infty} e^{-t \sqrt{2 n+2 k+1}} a_{n}^{k}(f) \frac{\theta(n, k)}{\sqrt{2 n+2 k+1}} h_{n-1}^{k}(x), \\
& f_{k}^{-}(t, x)=\sum_{n=0}^{+\infty} e^{-t \sqrt{2 n+2 k+1}} a_{n}^{k}(f) \frac{\theta(n+1, k)}{\sqrt{2 n+2 k+1}} h_{n+1}^{k}(x) .
\end{aligned}
$$

Remark 2. The same arguments used for the heat-diffusion integral show that $f_{k}^{ \pm}(t, x) \in$ $C^{\infty}\left(\mathbb{R}_{+} \times \mathbb{R}\right)$ and satisfy the differential-difference equations

$$
\begin{aligned}
& (i) \quad\left(L_{k, x}+\frac{\partial^{2}}{\partial t^{2}}\right) f_{k}^{ \pm}(t, x)= \pm 2 f_{k}^{ \pm}(t, x), \\
& (i i) \quad\left(T_{k, x} \pm x\right) F_{k}(f)(t, x)=\mp \frac{\partial}{\partial t} f_{k}^{ \pm}(t, x),
\end{aligned}
$$

where $F_{k}(f)(t, x)$ is the Poisson integral of $f$.

We now use (19) to find an integral formula for $f_{k}^{ \pm}(t, x)$. Using the subordination formula (7), taking $\beta=t \sqrt{2 n+2 k+1}$, making the change of variables $s \longrightarrow(2 n+2 k+1) u$, and then substituting $r=e^{-2 u}$ leads to the formula

$$
e^{-t \sqrt{2 n+2 k+1}}=\int_{0}^{1} L(t, r) r^{n+k+\frac{1}{2}} d r
$$

where

$$
L(t, r)=\frac{t e^{\frac{t^{2}}{2 \log r}}}{(2 \pi)^{\frac{1}{2}} r(-\log r)^{\frac{3}{2}}} .
$$

Then if $A_{k}(t, x, y)$ denotes the Poisson kernel (6), we have

$$
\begin{aligned}
A_{k}(t, x, y) & =\sum_{n=0}^{+\infty} e^{-t \sqrt{2 n+2 k+1}} h_{n}^{k}(x) h_{n}^{k}(y)=\sum_{n=0}^{+\infty} h_{n}^{k}(x) h_{n}^{k}(y) \int_{0}^{1} L(t, r) r^{n+k+\frac{1}{2}} d r \\
& =\int_{0}^{1} \sum_{n=0}^{+\infty} r^{n} h_{n}^{k}(x) h_{n}^{k}(y) L(t, r) r^{k+\frac{1}{2}} d r=\int_{0}^{1} L(t, r) U_{k}(r, x, y) r^{k+\frac{1}{2}} d r .
\end{aligned}
$$

Combining this and (1) we obtain

$$
\begin{aligned}
\left(T_{k, x}\right. & +x) A_{k}(t, x, y) \\
& =\frac{\sqrt{2}}{\sqrt{\pi}} c_{k} e^{-\frac{1}{2}\left(x^{2}+y^{2}\right)} \int_{0}^{1} \frac{(y-r x) t e^{\frac{t^{2}}{2 \log r}} e^{-\frac{r^{2} x^{2}+r^{2} y^{2}}{1-r^{2}}}}{(-\log r)^{\frac{3}{2}}\left(1-r^{2}\right)^{k+\frac{3}{2}}} E_{k}\left(\frac{2 r x}{1-r^{2}}, y\right) r^{k+\frac{1}{2}} d r .
\end{aligned}
$$

Now

$$
\left(T_{k, x}+x\right) F_{k}(f)(t, x)=\int_{\mathbb{R}}\left(T_{k, x}+x\right) A_{k}(t, x, y) f(y)|y|^{2 k} d y .
$$


From Propositions 1 and 2 , it is easy to check that $f_{k}^{+}(t, x) \longrightarrow 0$ as $t \longrightarrow+\infty$ and so

$$
f_{k}^{+}(t, x)=-\int_{t}^{+\infty} \frac{\partial}{\partial t} f_{k}^{+}(u, x) d u .
$$

Using (21), (20) and (19) we find after integration

$$
f_{k}^{+}(t, x)=\int_{\mathbb{R}} Q_{k}(t, x, y) f(y)|y|^{2 k} d y
$$

where

$$
Q_{k}(t, x, y)=e^{-\frac{1}{2}\left(x^{2}+y^{2}\right)} Q_{1, k}(t, x, y)
$$

and

$$
Q_{1, k}(t, x, y)=\int_{0}^{1} \frac{(y-r x)}{\left(1-r^{2}\right)^{k+2}} e^{-\frac{r^{2} x^{2}+r^{2} y^{2}}{1-r^{2}}} E_{k}\left(\frac{2 r x}{1-r^{2}}, y\right) W_{1, k}(t, r) d r
$$

with

$$
W_{1, k}(t, r)=\frac{\sqrt{2}}{\sqrt{\pi}} c_{k}\left(\frac{1-r^{2}}{-\log r}\right)^{\frac{1}{2}} e^{\frac{t^{2}}{2 \log r}} r^{k+\frac{1}{2}} .
$$

We now use (19) to find an integral formula for $f_{k}^{-}(t, x)$

$$
\begin{aligned}
\left(T_{k, x}\right. & -x) A_{k}(t, x, y) \\
& =\frac{\sqrt{2}}{\sqrt{\pi}} c_{k} e^{-\frac{1}{2}\left(x^{2}+y^{2}\right)} \int_{0}^{1} \frac{(r y-x) t e^{\frac{t^{2}}{2 \log r}} e^{-\frac{r^{2} x^{2}+r^{2} y^{2}}{1-r^{2}}}}{(-\log r)^{\frac{3}{2}}\left(1-r^{2}\right)^{k+\frac{3}{2}}} E_{k}\left(\frac{2 r x}{1-r^{2}}, y\right) r^{k-\frac{1}{2}} d r .
\end{aligned}
$$

Now

$$
\left(T_{k, x}-x\right) F_{k}(f)(t, x)=\int_{\mathbb{R}}\left(T_{k, x}-x\right) A_{k}(t, x, y) f(y)|y|^{2 k} d y .
$$

The same reasoning as above gives $f_{k}^{-}(t, x) \longrightarrow 0$ as $t \longrightarrow+\infty$ and so

$$
f_{k}^{-}(t, x)=-\int_{t}^{+\infty} \frac{\partial}{\partial t} f_{k}^{-}(u, x) d u .
$$

Using (23), (22) and (19) we find after integration

$$
f_{k}^{-}(t, x)=\int_{\mathbb{R}} M_{k}(t, x, y) f(y)|y|^{2 k} d y,
$$

where

$$
M_{k}(t, x, y)=e^{-\frac{1}{2}\left(x^{2}+y^{2}\right)} M_{1, k}(t, x, y)
$$

and

$$
M_{1, k}(t, x, y)=\int_{0}^{1} \frac{(x-r y) e^{-\frac{r^{2} x^{2}+r^{2} y^{2}}{1-r^{2}}}}{\left(1-r^{2}\right)^{k+2}} E_{k}\left(\frac{2 r x}{1-r^{2}}, y\right) Y_{k}(t, r) d r,
$$

with

$$
Y_{k}(t, r)=\frac{\sqrt{2}}{\sqrt{\pi}} c_{k}\left(\frac{1-r^{2}}{-\log r}\right)^{\frac{1}{2}} e^{\frac{t^{2}}{2 \log r}} r^{k-\frac{1}{2}}
$$


Theorem 7. There exists a positive constant $C$ such that for $1<p<+\infty, f \in L^{p}\left(\mathbb{R},|x|^{2 k} d x\right)$, we have

$$
\left\|f_{k}^{ \pm}(t, \cdot)\right\|_{k, p} \leq C e^{-t \sqrt{2 k+1}}\|f\|_{k, p} .
$$

Proof. We have

$$
\left\|f_{k}^{ \pm}(t, \cdot)\right\|_{k, p}=\left\| \pm \mathcal{H}_{k}^{ \pm} F_{k}(f)(t, \cdot)\right\|_{k, p} \leq C\left\|F_{k}(f)(t, \cdot)\right\|_{k, p} \leq C e^{-t \sqrt{2 k+1}}\|f\|_{k, p} .
$$

Theorem 8. There exists a positive constant $C$ such that for $f \in L^{1}\left(\mathbb{R},|x|^{2 k} d x\right)$, we have

$$
\sup _{\lambda>0}\left(\lambda \int_{\left\{x \in \mathbb{R}:\left|f_{k}^{ \pm}(t, x)\right|>\lambda\right\}} d x\right) \leq C e^{-t \sqrt{2 k+1}}\|f\|_{k, 1} .
$$

Proof. We have

$$
\begin{aligned}
\sup _{\lambda>0}\left(\lambda \int_{\left\{x \in \mathbb{R}:\left|f_{k}^{ \pm}(t, x)\right|>\lambda\right\}} d x\right) & =\sup _{\lambda>0}\left(\lambda \int_{\left\{x \in \mathbb{R}:\left| \pm \mathcal{H}_{k}^{ \pm} F_{k}(f)(t, x)\right|>\lambda\right\}} d x\right) \\
& \leq C\left\|F_{k}(f)(t, \cdot)\right\|_{k, 1} \leq C e^{-t \sqrt{2 k+1}}\|f\|_{k, 1} .
\end{aligned}
$$

\section{Acknowledgments}

The authors thank the anonymous referees for their careful reading of the manuscript and their valuable suggestions to improve the style of this paper.

\section{References}

[1] de Jeu M.F.E., The Dunkl transform, Invent. Math. 113 (1993), 147-162.

[2] Dunkl C.F., Hankel transforms associated to finite reflection groups, in Hypergeometric Functions on Domains of Positivity, Jack Polynomials and Applications (Tampa, 1991), Contemp. Math. 138 (1992), 123138.

[3] El Garna A., The left-definite spectral theory for the Dunkl-Hermite differential-difference equation, J. Math. Anal. Appl. 298 (2004), 463-486.

[4] Gosselin J., Stempak K., Conjugate expansions for Hermite functions, Illinois J. Math. 38 (1994), $177-197$.

[5] Journé J.-L., Calderón-Zygmund operators, pseudo-differential operators and the Cauchy integral of Calderón, Lecture Notes in Mathematics, Vol. 994, Springer-Verlag, Berlin, 1983.

[6] Nowak A., Stempak K., Riesz transforms for the Dunkl harmonic oscillator, Math. Z., to appear, arXiv:0802.0474.

[7] Rosenblum M., Generalized Hermite polynomials and the Bose-like oscillator calculus, in Nonselfadjoint Operators and Related Topics (Beer Sheva, 1992), Oper. Theory Adv. Appl., Vol. 73, Birkhäuser, Basel, 1994, 369-396.

[8] Rösler M., Generalized Hermite polynomials and the heat equation for Dunkl operators, Comm. Math. Phys. 192 (1998), 519-542, q-alg/9703006.

[9] Rösler M., Positivity of Dunkl's intertwining operator, Duke Math. J. 98 (1999), 445-463, q-alg/9710029.

[10] Stein E.M., Topics in harmonic analysis related to the Littlewood-Paley theory, Annals of Mathematics Studies, no. 63, Princeton University Press, Princeton, NJ, 1970.

[11] Stempak K., Torrea J.L., Poisson integrals and Riesz transforms for Hermite function expansions with weights, J. Funct. Anal. 202 (2003), 443-472.

[12] Thangavelu S., Lectures on Hermite and Laguerre expansions, Mathematical Notes, Vol. 42, Princeton University Press, Princeton, NJ, 1993. 